\title{
Canonical-covariant Wigner function in polar form
}

\author{
T. Hakioğlu \\ Department of Physics, Bilkent University, 06533 Ankara, Turkey, and High Energy Physics Division, \\ Argonne National Laboratories, Argonne, Illinois 60439-4815
}

Received March 21, 2000; revised manuscript received August 30, 2000; accepted September 5, 2000

The two-dimensional Wigner function is examined in polar canonical coordinates, and covariance properties under the action of affine canonical transformations are derived. (C) 2000 Optical Society of America [S0740-3232(00)03912-0]

OCIS codes: $080.0080,070.0070,270.0270,000.3860,000.1600$.

\section{INTRODUCTION}

Phase space is a remarkable concept facilitating the generalized understanding of the transition between the classical and the quantum formulations and is principally built on proper sets of independent dynamical variables (the canonical coordinates) describing the considered physical system and symmetry transformations between them. The transformations induced on the phase space are said to be canonical if the equations of motion are form invariant under their action. Although the canonical form derives its name from Hamilton for his historical work on the time evolution of quadratic systems, a general frame on which a canonical structure can be built is independent from any dynamical system considered. One simple feature of these systems initially considered by Hamilton is that the preservation of the canonical structure becomes identical to the covariance under time evolution. Indeed, the simplicity afforded by quadratic Hamiltonians is that they provide a natural transition between the linear (ray) optics and the phase-space representation in mechanics in terms of the standard canonical phase-space pair, viz., linear coordinate and momentum. If one replaces the time with the parameter defined along the optical axis, the equations of motion obtained for the phase-space variables are identical in terms of mechanics to those in the linear optics. The importance of the quadratic Hamiltonians is not limited by the classical linear optics correspondence and extends far beyond the classical realm into the quantum world. The classical and the quantum versions of a quadratic system respect the same phase-space symmetry transformations, viz., affine canonical transformations (ACT's). For these systems, the equations of motion for the classical phase-space pair $(p, q)$ and their quantum counterparts $(\hat{p}, \hat{q})$ are identical.

One of the most important conceptual breakthroughs in the phase-space representations of quantum systems was made by Weyl ${ }^{1}$ in 1927 and by Wigner ${ }^{2}$ in 1932 and later by Groenewold ${ }^{3}$ in 1946 and by $\mathrm{Moyal}^{3}$ in 1949. The Weyl-Wigner-Groenewold-Moyal (WWGM) correspondence is based on the existence of an orthogonal and complete operator basis [the Weyl-Heisenberg (WH) basis] in which an arbitrary operator $F(\hat{p}, \hat{q})$ as a function of the canonical phase-space operators $(\hat{p}, \hat{q})$ can be invertibly mapped to a classical phase-space function $f(p, q)$. The crucial property is that, if the basis operators are symmetrically ordered, the WWGM correspondence is covariant under the action of ACT's between the transformed operator $F^{\prime}(\hat{p}, \hat{q}) \equiv F\left(\hat{p}^{\prime}, \hat{q}^{\prime}\right)$ and its transformed symbol $f^{\prime}(p, q) \equiv f\left(p^{\prime}, q^{\prime}\right)$, where the phase-space variables $(p, q)$ and the phase-space operators $(\hat{p}, \hat{q})$ are transformed under the same linear map.

The representations in quantum-mechanical phase space and the distribution functions studied therein were largely limited until very recently to the linear canonical coordinate and momentum $(p, q)$. The Wigner function $W_{\psi}(p, q)$, as the best example for such representations, is well known to be covariant under ACT's, and its time evolution under quadratic Hamiltonians is given by the classical Liouville equation. By contrast, the action of the ACT's on the linear canonical phase-space pair $(p, q)$ consists in the symmetry operations for such systems, whose dynamics are governed by quadratic Hamiltonians. This implies that the time dependence of the Wigner function in quadratic systems can be represented by timeparameterized trajectories that coincide with the classical ones. This seems to be the ultimate limit to which one can push the classical-quantum correspondence in the phase space. If we bear in mind that standard quadratic systems represented in the linear canonical coordinate and momentum are not that numerous or that they are Gaussian approximations to the original ones, the utility of these results is limited.

The general canonical phase-space formulation of any mechanical system (whether quantum or classical) is expected to be independent of the choice of a particular canonical basis, which suggests that there can be more than one such basis doing the same job. In some cases a nonlinear canonical transformation generated by, say, $W_{\infty}$ can be used to connect these two bases. It can be shown that $^{4}$ one WWGM correspondence scheme transforms noncovariantly to another such scheme under a general nonlinear canonical transformation. Within a particular correspondence scheme the Weyl map is then expected to 
maintain the covariance under ACT's acting on the canonical basis of the choice. In this paper we will consider the covariant phase-space formulation of two-dimensional (2D) systems in polar space coordinates and their canonical momenta. The spectrum of the radial phase-space operator is shown to be the logarithmic variate of the classical one, $r \in \mathbb{R}^{+}$, and in the operator language these two representations are connected by a Fourier-Mellin transformation. The logarithmic radial (log-radial) coordinate is itself a representation of $r \in \mathbb{R}^{+}$in Cartesian $r \in \mathbb{R}$, enabling a radial WWGM quantization as well as the corresponding radial Wigner function formulations through the standard Cartesian formalism. It must be emphasized that, although the polar coordinate basis is the most natural choice for systems with specific rotational symmetries, the formulation is not limited in applications to them.

Section 2 is devoted to the polar representation of the Wigner function based on the log-radial spectrum. Subsection 2.A discusses the log-radial and the angular canonical bases and presents the respective Wigner functions. In each part therein, the properties of the Wigner function are examined, and the covariance under ACT's is discussed. There I also define a nonlinear canonical transformation that basically undoes the effect of the logradial spectrum at the expense of losing most of the covariances of the Wigner function. In Subsection 2.B the log-radial and the angular bases are combined in a product form, and the polar Wigner function is introduced. Section 3 is a short and elementary example of the polar Wigner function. The validity of the canonical formalism presented here in generic mechanical as well as optical systems is implied by the absence of $\hbar$ or by the reduced wavelength $\Varangle$ throughout the study.

\section{CONTINUOUS POLAR REPRESENTATION}

Let us assume a $2 \mathrm{D}$ wave field in the $x-y$ plane, with $z$ representing the evolution parameter of the wave along the optical axis. If we follow the wave along the instantaneous direction of propagation by a screen normal to that direction, the wave field at the particular location $z$ of the screen can be given by

$$
\Psi(r, \phi ; z)=\sum_{n \in \mathbb{Z}} \widetilde{\Psi}_{n}(r ; z) \exp (i n \phi) .
$$

Throughout the paper we will assume that the screen location is fixed. We will hence consider the $z$ coordinate as implicit in all expressions.

\section{A. Polar Canonical Basis}

Our main aim in this section is to introduce displacement operators in polar representation in the form of an orthogonal and complete operator basis for the $2 \mathrm{D} \mathrm{WH}$ group of polar canonical operators. The standard $2 \mathrm{D}$ Wigner function $W_{\Psi}(\mathbf{p}, \mathbf{q})$, where $\mathbf{p}=\left(p_{x}, p_{y}\right)$ and $\mathbf{q}$ $=\left(q_{x}, q_{y}\right)$ are the canonical phase-space variables of the linear momentum and coordinate in the independent $x$ and $y$ directions, respectively, is written in terms of a complete and orthogonal operator basis,

$$
\hat{\Delta}(\mathbf{p}, \mathbf{q})=\hat{\Delta}_{x}\left(p_{x}, q_{x}\right) \otimes \hat{\Delta}_{y}\left(p_{y}, q_{y}\right),
$$

in a representation-independent manner as

$$
W_{\Psi}(\mathbf{p}, \mathbf{q})=\langle\Psi, \hat{\Delta},(\mathbf{p}, \mathbf{q}) \Psi\rangle .
$$

The operator bases $\hat{\Delta}_{i}$, where $i=x, y$, are given by the unitary displacement operator (WH basis) $\hat{D}_{\alpha_{i}, \beta_{i}}$ as

$$
\begin{aligned}
\hat{\Delta}_{i} & =\hat{\Delta}\left(p_{i}, q_{i}\right) \\
& =\int_{\mathbb{R}} \frac{\mathrm{d} \alpha_{i}}{2 \pi} \int_{\mathbb{R}} \frac{\mathrm{d} \beta_{i}}{2 \pi} \exp \left[i\left(\alpha_{i} q_{i}-\beta_{i} p_{i}\right)\right] \hat{D}_{\alpha_{i}, \beta_{i}}, \\
\hat{D}_{\alpha_{i}, \beta_{i}} & =\exp \left[i\left(\alpha_{i} \hat{q}_{i}+\beta_{i} \hat{p}_{i}\right)\right],
\end{aligned}
$$

where $\hat{q}_{i}, \hat{p}_{i}$ are the canonical linear coordinate and momentum operators satisfying $\left[\hat{q}_{i}, \hat{p}_{j}\right]=i \delta_{i, j}$ and $\alpha_{i}, \beta_{i}$; $q_{i}, p_{i} \in \mathbb{R}$ for $i=x, y$. The properties of the standard Wigner function in two degrees of freedom given by Eq. (3) are well known and have been examined in great detail in a large number of publications. ${ }^{5}$

Our aim is to develop a covariant formalism for the 2D Wigner function represented in terms of the polar canonical momentum and coordinates $p_{r}, v_{r} ; p_{\theta}, v_{\theta}$, where, respectively, $p_{r}, v_{r}$ are the radial and $p_{\theta}, v_{\theta}$ are the angular canonical momentum and coordinate pairs. In our case the domain of the radial phase-space variables is $-\infty<v_{r}, p_{r},<\infty$, and the angular ones are $p_{\theta} \in \mathbb{Z}$ and $-\pi \leqslant v_{\theta}<\pi$. The formalism will be based on a direct product form similar to that of Eq. (2) but in terms of the radial $\hat{\Delta}_{r}\left(p_{r}, v_{r}\right)$ and angular $\hat{\Delta}_{\theta}\left(p_{\theta}, v_{\theta}\right)$ operator bases.

\section{Radial Part}

A log-radial Wigner function based on the idea of Dirac's self-adjoint radial momentum operator ${ }^{6} \hat{p}_{r}$ was recently proposed. $^{7}$ In these studies the radial momentum operator $\hat{p}_{r}$ in the radial $(r)$ coordinate representation is written by

$$
\hat{p}_{r} \rightarrow-i\left(r \frac{\partial}{\partial r}+\eta\right), \quad \eta \in \mathbb{R} .
$$

We will see below that $\eta$ is related to the dimensionality of the space. For the radial representations in a $d$-dimensional space, we have $\eta=d / 2$.

If we write the radial position operator $\hat{v}_{r}=\ln \hat{r}$, where $\hat{v}_{r} \rightarrow \ln r$, in the radial coordinate representation, the Dirac commutator of $\hat{v}_{r}$ and $\hat{p}_{r}$ yields

$$
\left[\hat{v}_{r}, \hat{p}_{r}\right]=i
$$

The eigenspace of $\hat{p}_{r}$ is spanned by

$$
\varphi_{\lambda}(r)=(1 / \sqrt{2 \pi}) r^{i \lambda-\eta},
$$

where $\lambda$ is the radial momentum eigenvalue and, for $\lambda \in \mathrm{R}, \varphi_{\lambda}(r)$ is a complete and orthogonal basis for the harmonic analysis on the positive half-plane, viz., generalized positive Mellin transform. ${ }^{8,9}$ The function space is a Hilbert space defined by the inner product

$$
\langle\psi, \phi\rangle_{r} \equiv \int_{0}^{\infty} \mathrm{d} r r^{2 \eta-1} \psi^{*}(r) \phi(r), \quad \psi, \phi \in \mathcal{L}_{2}^{(\eta)}\left(\mathbb{R}^{+}\right)
$$

and by the dual orthogonality relations 


$$
\begin{gathered}
\left\langle\varphi_{\lambda^{\prime}}, \varphi_{\lambda}\right\rangle_{r}=\int_{0}^{\infty} \mathrm{d} r r^{2 \eta-1} \varphi_{\lambda^{\prime}} *(r) \varphi_{\lambda}(r)=\delta\left(\lambda^{\prime}-\lambda\right), \\
\int_{-\infty}^{\infty} \mathrm{d} \lambda \varphi_{\lambda} *(r) \varphi_{\lambda}\left(r^{\prime}\right)=\delta\left(r-r^{\prime}\right) r^{-2 \eta+1} .
\end{gathered}
$$

In Eq. (8), $\mathcal{L}_{2}^{(\eta)}\left(\mathbb{R}^{+}\right)$denotes the Hilbert space of functions with a finite norm, i.e., $\|\psi\|_{2} \equiv\langle\psi, \psi\rangle_{r}<\infty$. It can be directly verified that $\hat{p}_{r}$ is self-adjoint in $\mathcal{L}_{2}^{(\eta)}\left(\mathrm{R}^{+}\right)$over the inner product defined by Eq. (8). In other words,

$$
\left\langle\psi, \hat{p}_{r} \phi\right\rangle_{r}=\left\langle\hat{p}_{r} \psi, \phi\right\rangle_{r}-\left.i \psi^{*}(r) r^{2 \eta} \phi(r)\right|_{0} ^{\infty},
$$

where, for all functions in $\mathcal{L}_{2}^{(\eta)}\left(\mathrm{R}^{+}\right)$, the last term in Eq. (11) vanishes, and hence $\hat{p}_{r}$ is self-adjoint. A specific case of Eqs. (9) and (10) is $\eta=1 / 2$, which corresponds to the one-dimensional case in which the weight factors due to $r \rightarrow \ln r$ vanish and the basis in Eq. (7) becomes an isomorphic map between $R$ and its nonnegative part $\mathbb{R}^{+}$, which is a more standard version of the Mellin transformation. ${ }^{9}$ Using the inner product in Eq. (8) and the orthogonality relations in Eqs. (9) and (10), we can expand an arbitrary function $\psi(r)$ in $\mathcal{L}_{2}^{(\eta)}\left(\mathbb{R}^{+}\right)$in the Mellin basis as

$$
\psi(r)=\int_{-\infty}^{\infty} \mathrm{d} \lambda A(\lambda) \varphi_{\lambda}(r), \quad A(\lambda)=\left\langle\varphi_{\lambda}, \psi\right\rangle_{r}
$$

The inner product defined by Eq. (8) can be expressed in the radial momentum- $\lambda$ representation as

$$
\langle\psi, \phi\rangle_{r}=\int_{-\infty}^{\infty} \mathrm{d} \lambda A^{*}(\lambda) B(\lambda), \quad A(\lambda), B(\lambda) \in \mathcal{L}_{2}(\mathbb{R}),
$$

where $\mathcal{L}_{2}(\mathbb{R})$ is the usual Hilbert space of squareintegrable functions on the real line. In close analogy with Eqs. (4), the radial canonical operator basis can now be established as

$$
\begin{aligned}
\hat{\Delta}_{r}\left(p_{r}, v_{r}\right)= & \int_{-\infty}^{\infty} \frac{\mathrm{d} \alpha_{r}}{2 \pi} \int_{-\infty}^{\infty} \frac{\mathrm{d} \beta_{r}}{2 \pi} \\
& \times \exp \left[-i\left(\alpha_{r} v_{r}+\beta_{r} p_{r}\right)\right] \hat{D}_{r}\left(\alpha_{r}, \beta_{r}\right), \\
\hat{D}_{r}\left(\alpha_{r}, \beta_{r}\right)= & \exp \left[i\left(\alpha_{r} \hat{v}_{r}+\beta_{r} \hat{p}_{r}\right)\right],
\end{aligned}
$$

where $v_{r}, p_{r} \in \mathrm{R}$ are the log-radial phase-space variables. The properties of the log-radial canonical basis $\hat{\Delta}_{r}\left(p_{r}, v_{r}\right)$ follow from those of $\hat{D}_{r}\left(\alpha_{r}, \beta_{r}\right)$, which are

$$
\begin{aligned}
\hat{D}_{r}(0,0)= & \hat{\mathbb{I}}, \\
\hat{D}_{r}^{\dagger}\left(\alpha_{r}, \beta_{r}\right)= & \hat{D}_{r}^{-1}\left(\alpha_{r}, \beta_{r}\right)=\hat{D}_{r}\left(-\alpha_{r},-\beta_{r}\right), \\
\operatorname{Tr}\left\{\hat{D}_{r}\left(\alpha_{r}, \beta_{r}\right)\right\}= & 2 \pi \delta\left(\alpha_{r}\right) \delta\left(\beta_{r}\right), \\
\hat{D}_{r}\left(\alpha_{r}, \beta_{r}\right) \hat{D}_{r}\left(\alpha_{r}^{\prime}, \beta_{r}^{\prime}\right) & \\
= & \exp \left[-i\left(\alpha_{r} \beta_{r}^{\prime}-\beta_{r} \alpha_{r}^{\prime}\right) / 2\right] \\
& \times \hat{D}_{r}\left(\alpha_{r}+\alpha_{r}^{\prime}, \beta_{r}+\beta_{r}^{\prime}\right) .
\end{aligned}
$$

Equation (15) defines the unit element. Equation (16) is the statement of unitarity and inversion guaranteed by the self-adjointness of $\hat{p}_{r}$ and $\hat{v}_{r}$ over the inner product in
Eqs. (9) and (10). The orthogonality of the basis is guaranteed by Eq. (17), where Tr stands for the trace as obtained by

$$
\operatorname{Tr}\left\{\hat{D}_{r}\left(\alpha_{r}, \beta_{r}\right)\right\} \equiv \int_{-\infty}^{\infty} \mathrm{d} \lambda\left\langle\varphi_{\lambda}, \hat{D}_{r}\left(\alpha_{r}, \beta_{r}\right) \varphi_{\lambda}\right\rangle_{r}
$$

The composition law is stated by Eq. (18). These properties are translated for $\hat{\Delta}_{r}\left(p_{r}, v_{r}\right)$ as

$$
\begin{aligned}
\hat{\Delta}_{r}^{\dagger}\left(p_{r}, v_{r}\right) & =\hat{\Delta}_{r}\left(p_{r}, v_{r}\right), \\
\operatorname{Tr}\left\{\hat{\Delta}_{r}\left(p_{r}, v_{r}\right)\right\} & =\frac{1}{2 \pi} \\
\operatorname{Tr}\left\{\hat{\Delta}_{r}\left(p_{r}, v_{r}\right) \hat{\Delta}_{r}\left(p_{r}^{\prime}, v_{r}^{\prime}\right)\right\} & =\frac{1}{2 \pi} \delta\left(p_{r}-p_{r}^{\prime}\right) \delta\left(v_{r}-v_{r}^{\prime}\right) \\
\int_{-\infty}^{\infty} \mathrm{d} v_{r} \int_{-\infty}^{\infty} \mathrm{d} p_{r} \hat{\Delta}_{r}\left(p_{r}, v_{r}\right) & =\hat{\mathbb{I}} \\
\int_{-\infty}^{\infty} \mathrm{d} v_{r} \hat{\Delta}_{r}\left(p_{r}, v_{r}\right) & =\hat{\widetilde{\mathcal{P}}}_{r}\left(p_{r}\right) \\
\int_{-\infty}^{\infty} \mathrm{d} p_{r} \hat{\Delta}_{r}\left(p_{r}, v_{r}\right) & =\hat{\mathcal{P}}_{r}\left(v_{r}\right),
\end{aligned}
$$

where $\hat{\mathcal{P}}_{r}$ and $\widetilde{\widetilde{\mathcal{P}}}_{r}$ are the radial projection operators as defined by

$$
\begin{aligned}
& \hat{\mathcal{P}}_{r}\left(v_{r}\right) \hat{\mathcal{P}}_{r}\left(v_{r}^{\prime}\right)=\delta\left(v_{r}-v_{r}^{\prime}\right) \hat{\mathcal{P}}_{r}\left(v_{r}\right), \\
& \int \mathrm{d} v_{r} \hat{\mathcal{P}}_{r}\left(v_{r}\right)=\hat{\mathbb{I}}, \\
& \hat{\mathcal{P}}_{r}\left(p_{r}\right) \hat{\mathcal{P}}_{r}\left(p_{r}^{\prime}\right)=\delta\left(p_{r}-p_{r}^{\prime}\right) \hat{\mathcal{P}}_{r}\left(p_{r}\right), \\
& \int \mathrm{d} p_{r} \hat{\widetilde{\mathcal{P}}}_{r}\left(p_{r}\right)=\hat{\mathbb{I}},
\end{aligned}
$$

with

$$
\begin{aligned}
&\left\langle\psi, \hat{\mathcal{P}}_{r}\left(v_{r}\right) \phi\right\rangle_{r} \equiv \exp \left(2 \eta v_{r}\right) \psi^{*}\left(\exp v_{r}\right) \phi\left(\exp v_{r}\right), \\
&\left\langle\psi, \hat{\widetilde{P}}_{r}\left(p_{r}\right) \phi\right\rangle_{\lambda} \equiv A^{*}\left(p_{r}\right) B\left(p_{r}\right),
\end{aligned}
$$

where $A\left(p_{r}\right)$ and $B\left(p_{r}\right)$ are the Mellin transforms of $\psi(r)$ and $\phi(r)$ as calculated by Eq. (12).

Log-radial Wigner function and its properties. The representation-independent form of the log-radial Wigner function for a state $\psi$ will be defined as

$$
W_{\psi}\left(p_{r}, v_{r}\right)=\left\langle\psi, \hat{\Delta}_{r}\left(p_{r}, v_{r}\right) \psi\right\rangle_{r} .
$$

Equation (29) is represented in the radial coordinate basis as

$$
\begin{aligned}
W_{\psi}\left(p_{r}, v_{r}\right)= & \frac{1}{2 \pi} \int_{-\infty}^{\infty} \mathrm{d} \beta_{r} \exp \left(-i \beta_{r} p_{r}\right) \exp \left(2 \eta v_{r}\right) \\
& \times \psi^{*}\left[\exp \left(v_{r}+\beta_{r} / 2\right)\right] \psi\left[\exp \left(v_{r}-\beta_{r} / 2\right)\right]
\end{aligned}
$$

and in the radial momentum basis as 


$$
\begin{aligned}
W_{A_{\psi}}\left(p_{r}, v_{r}\right)= & \frac{1}{2 \pi} \int_{-\infty}^{\infty} \mathrm{d} \alpha_{r} \exp \left(i \alpha_{r} v_{r}\right) A^{*} \\
& \times\left(p_{r}+\alpha_{r} / 2\right) A\left(p_{r}-\alpha_{r} / 2\right) .
\end{aligned}
$$

The static properties of the radial Wigner function follow directly from Eqs. (29)-(31). These are

(1) $W_{\psi}\left(p_{r}, v_{r}\right)$ is real, namely,

$$
W_{\psi}\left(p_{r}, v_{r}\right)=W_{\psi} *\left(p_{r}, v_{r}\right)
$$

which follows directly from Eq. (20).

(2) The integral of $W_{\psi}\left(p_{r}, v_{r}\right)$ with respect to one of the phase-space variables yields the marginal probability with respect to the other variable:

$$
\begin{aligned}
\int_{-\infty}^{\infty} \mathrm{d} v_{r} W_{\psi}\left(p_{r}, v_{r}\right) & =\left\langle\psi, \hat{\widetilde{\mathcal{P}}}\left(p_{r}\right) \psi\right\rangle_{r}=\left|A\left(p_{r}\right)\right|^{2}, \\
\int_{-\infty}^{\infty} \mathrm{d} p_{r} W_{\psi}\left(p_{r}, v_{r}\right) & =\left\langle\psi, \hat{\mathcal{P}}\left(v_{r}\right) \psi\right\rangle_{r} \\
& =\exp \left(2 \eta v_{r}\right)\left|\psi\left(\exp v_{r}\right)\right|^{2},
\end{aligned}
$$

which follow directly from Eqs. (24) and (25).

(3) Static covariance properties: The standard $p, q$ Wigner function is known to be covariant under ACT's. For a system with one degree of freedom, the ACT is a five parameter group of which three are the parameters of the group of linear canonical transformations (LCT's). The remaining two are the parameters of the Galilean transformations. A similar construction can also be made for the log-radial Wigner function. Below we will examine the covariance under ACT's within each subgroup independently. In paragraphs (a) and (b) the log-radial analogs of the Galilean transformations will be studied; in paragraphs (c)-(e) the LCT's will be studied.

Radial analogs of the Galilean transformations. These are as follows.

(a) Covariance under radial dilations. We define a map from a wave function $\psi(r)$ to $\psi^{\prime}(r)$ by

$$
\psi^{\prime}(r) \equiv \exp \left(i \beta_{r}^{\prime} \hat{p}_{r}\right) \psi(r)=\exp \left(\eta \beta_{r}^{\prime}\right) \psi\left[\exp \left(\beta_{r}^{\prime}\right) r\right] .
$$

Inserting Eq. (33) into Eqs. (29) and (30), we find that

$$
W_{\psi}\left(p_{r}, v_{r}\right)=W_{\psi^{\prime}}\left(p_{r}, v_{r}+\beta_{r}^{\prime}\right),
$$

which states the covariance of the Wigner function under radial dilations in Eq. (35).

(b) Covariance under local phase shifts. We now define a map from $\psi(r)$ to $\psi^{\prime}(r)$ as

$$
\psi^{\prime}(r) \equiv \exp \left(-i \alpha_{r}^{\prime} \hat{v}_{r}\right) \psi(r)=r^{-i \alpha_{r}^{\prime}} \psi(r) .
$$

Inserting Eq. (37) into Eqs. (29) and (30), we find that

$$
W_{\psi}\left(p_{r}, v_{r}\right)=W_{\psi^{\prime}}\left(p_{r}-\alpha_{r}^{\prime}, v_{r}\right),
$$

which states the covariance of the Wigner function under Galilean transformations on radial momentum $p_{r}$.

(c) Covariance under radial linear canonical transformations. A general LCT acting on the radial phase space $p_{r}, v_{r}$ will be defined by the map

$$
\left(\begin{array}{c}
p_{r}^{\prime} \\
v_{r}^{\prime}
\end{array}\right)=g\left(\begin{array}{l}
p_{r} \\
v_{r}
\end{array}\right), \quad g=\left(\begin{array}{ll}
a & b \\
c & d
\end{array}\right), \quad \operatorname{det} g=1,
$$

where $g$ is in the group $\operatorname{Sp}(2, \mathbb{R})$ of $2 \times 2$ symplectic matrices. The three one-parameter subgroups will be identified in the conventional way by

$$
\begin{aligned}
& g_{1}=\left[\begin{array}{cc}
\cos \sigma & -\sin \sigma \\
\sin \sigma & \cos \sigma
\end{array}\right], \quad g_{2}=\left[\begin{array}{cc}
\cosh \tau & -\sinh \tau \\
-\sinh \tau & \cosh \tau
\end{array}\right], \\
& g_{3}=\left[\begin{array}{cc}
\exp (-\chi) & 0 \\
0 & \exp (\chi)
\end{array}\right],
\end{aligned}
$$

with $-\pi \leqslant \sigma<\pi,-\infty<\tau<\infty$, and $-\infty<\chi<\infty$. We now examine the action of each subgroup by considering $g=g_{i}$ for $i=1,2,3$ independently. The representation $\hat{T}_{g}$ of the transformation in the operator basis $\hat{\Delta}_{r}$ is given by

$$
\hat{T}_{g}: \hat{\Delta}_{r}\left(p_{r}, v_{r}\right)=\hat{T}_{g} \hat{\Delta}_{r}\left(p_{r}, v_{r}\right) \hat{T}_{g}^{-1} \equiv \hat{\Delta}_{r}\left(p_{r}^{\prime}, v_{r}^{\prime}\right) .
$$

We expand $\hat{T}_{g}$ in the complete and orthogonal radial WH basis as

$$
\hat{T}_{g}=\int_{-\infty}^{\infty} \mathrm{d} \gamma_{r} \int_{-\infty}^{\infty} \mathrm{d} \delta_{r} C_{r}^{(g)}\left(\gamma_{r}, \delta_{r}\right) \hat{D}_{r}\left(\gamma_{r}, \delta_{r}\right),
$$

where the coefficients $C_{r}^{(g)}$ characterize the transformation. More generally, $\hat{D}_{r}$ (or, alternatively, its Fourier transform $\hat{\Delta}_{r}$ ) is an operator basis for any HilbertSchmidt operator. Using the unitarity of $\hat{D}_{r}$ 's as stated in Eq. (16) and demanding the unitarity of $\hat{T}_{g}$ 's we can derive a condition on the coefficients as $\left[C_{r}^{(g)}\left(\gamma_{r}, \delta_{r}\right)\right]^{*}$ $=C_{r}^{\left(g^{-1}\right)}\left(\gamma_{r}, \delta_{r}\right)$. Through Eq. (41) the coefficients also satisfy

$$
\begin{aligned}
C_{r}^{(g)}\left(\epsilon-\alpha_{r}, v-\beta_{r}\right) & \\
= & \exp \left\{i\left[\epsilon\left(\beta_{r}+\beta_{r}^{\prime}\right)-v\left(\alpha_{r}+\alpha_{r}^{\prime}\right)\right] / 2\right\} \\
& \times C_{g}^{(r)}\left(\epsilon-\alpha_{r}^{\prime}, v-\beta_{r}^{\prime}\right)
\end{aligned}
$$

for all $\epsilon, v, \alpha_{r}, \beta_{r}$, where

$$
\left(\begin{array}{c}
\alpha_{r}^{\prime} \\
\beta_{r}^{\prime}
\end{array}\right)=g\left(\begin{array}{c}
\alpha_{r} \\
\beta_{r}
\end{array}\right)
$$

Although a general solution to Eq. (44) can be given as

$$
C_{r}^{(g)}\left(\alpha_{r}, \beta_{r}\right)=\mathcal{N} \exp \left[i\left(U \alpha_{r}^{2}+V \beta_{r}^{2}+W \alpha_{r} \beta_{r}\right)\right],
$$

where $U, V, W$, and $\mathcal{N}$ are functions of the parameters of $\mathrm{g}$, it is more illuminating to give the solutions for each subgroup in Eqs. (40) separately. Using Eqs. (39) in Eq. (43), we find that

$$
\begin{aligned}
C_{r}^{\left(g_{1}\right)}\left(\alpha_{r}, \beta_{r}\right)= & \frac{\exp (i \pi / 2)}{4 \pi}[\sin (\sigma / 2)]^{-1} \\
& \times \exp \left[-(i / 4) \cot (\sigma / 2)\left(\alpha_{r}^{2}+\beta_{r}^{2}\right)\right], \\
C_{r}^{\left(g_{2}\right)}\left(\alpha_{r}, \beta_{r}\right)= & \frac{1}{4 \pi}|\sinh (\tau / 2)|^{-1} \\
& \times \exp \left[-(i / 4) \operatorname{coth}(\tau / 2)\left(\alpha_{r}^{2}-\beta_{r}^{2}\right)\right], \\
C_{r}^{\left(g_{3}\right)}\left(\alpha_{r}, \beta_{r}\right)= & \frac{1}{4 \pi}|\sinh (\chi / 2)|^{-1} \\
& \times \exp \left[-(i / 2) \operatorname{coth}(\chi / 2) \alpha_{r} \beta_{r}\right],
\end{aligned}
$$


where the normalizations are determined by the identity transformation limit such that

$$
\begin{gathered}
\lim _{g_{i} \rightarrow \mathrm{I}} C_{r}^{\left(g_{i}\right)}\left(\alpha_{r}, \beta_{r}\right)=\delta\left(\alpha_{r}\right) \delta\left(\beta_{r}\right), \\
\lim _{g_{i} \rightarrow \mathrm{I}} \hat{T}_{g_{i}}=\hat{\mathbb{I}}, \quad i=1,2,3 .
\end{gathered}
$$

It is also possible to show that a general group element can be obtained through $\hat{T}_{g} \equiv \hat{T}_{g_{3}} \hat{T}_{g_{2}} \hat{T}_{g_{1}}$, where $T_{g}$ is not exactly a group representation but a projective (ray) one ${ }^{9}$ satisfying $\hat{T}_{g} \hat{T}_{g^{\prime}}=\Lambda \hat{T}_{g g^{\prime}}$, where $\Lambda$ is an overall phase factor that depends on the parameters of $g, g^{\prime}$.

$T_{g}$ acts in the function space as a linear canonical integral transform. The expressions in the radial momentum representation are much simpler than those in the radial coordinate representations, which are defined by

$$
\hat{T}_{g} A\left(\lambda_{1}\right)=\int_{-\infty}^{\infty} \mathrm{d} \lambda_{2} c_{r}^{(g)}\left(\lambda_{1}, \lambda_{2}\right) A\left(\lambda_{2}\right)
$$

where the kernel of the integral transform $c_{r}^{(g)}$ can be found, for each subgroup $g_{i}(i=1,2,3)$, to be

$$
\begin{aligned}
c_{r}^{\left(g_{1}\right)}\left(\lambda_{1}, \lambda_{2}\right)= & \frac{\exp (i \pi / 4)}{\sqrt{2 \pi \sin \sigma}} \exp \left\{-\frac{i}{2 \sin \sigma}\right. \\
& \left.\times\left[\cos \sigma\left(\lambda_{1}{ }^{2}+\lambda_{2}^{2}\right)-2 \lambda_{1} \lambda_{2}\right]\right\}, \\
c_{r}^{\left(g_{2}\right)}\left(\lambda_{1}, \lambda_{2}\right)= & \frac{\exp (i \pi / 4)}{\sqrt{2 \pi \sinh \tau}} \exp \left\{-\frac{i}{2 \sinh \tau}\right. \\
& \left.\times\left[\cosh \tau\left(\lambda_{1}^{2}+\lambda_{2}^{2}\right)-2 \lambda_{1} \lambda_{2}\right]\right\}, \\
c_{r}^{\left(g_{3}\right)}\left(\lambda_{1}, \lambda_{2}\right)= & \exp (-\chi / 2) \delta\left[\lambda_{2}-\exp (-\chi) \lambda_{1}\right] .
\end{aligned}
$$

In Eqs. (49)-(51) the identity transformation is recovered in the appropriate limit as shown in Eq. (52). resentations of $\hat{p}_{r}$ and derive the infinitesimal generators in terms of $\hat{v}_{r}, \hat{p}_{r}$. Kernels such as those in Eqs. (49)(51) were studied in detail in Ref. 9. The generators of infinitesimal LCT are given by

$$
\begin{array}{ll}
\hat{T}_{g_{1}}=\exp \left(i 2 \sigma \hat{K}_{1}\right), & \hat{K}_{1}=\frac{1}{4}\left(\hat{p}_{r}^{2}+\hat{v}_{r}^{2}\right), \\
\hat{T}_{g_{2}}=\exp \left(i 2 \tau \hat{K}_{2}\right), & \hat{K}_{2}=\frac{1}{4}\left(\hat{p}_{r}^{2}-\hat{v}_{r}^{2}\right), \\
\hat{T}_{g_{3}}=\exp \left(i 2 \chi \hat{K}_{3}\right), & \hat{K}_{3}=\frac{1}{4}\left(\hat{p}_{r} \hat{v}_{r}+\hat{v}_{r} \hat{p}_{r}\right) .
\end{array}
$$

The action of the group elements $\hat{T}_{g_{i}}$ on the functions in $\mathcal{L}_{2}\left(R^{+}\right)$can now be very easily found, since the log-radial coordinate representations of the operators $\hat{K}_{i}$ are known. Their counterparts in terms of the linear momentum and coordinate are known in the theory of integral transforms ${ }^{9}$ and they define the $\mathrm{Sp}(2, \mathrm{R})$ algebra:

$$
\begin{aligned}
& {\left[\hat{K}_{1}, \hat{K}_{2}\right]=i \hat{K}_{3}, \quad\left[\hat{K}_{1}, \hat{K}_{3}\right]=-i \hat{K}_{2},} \\
& {\left[\hat{K}_{2}, \hat{K}_{3}\right]=-i \hat{K}_{1},}
\end{aligned}
$$

with the central element in this case being $\hat{K}^{2}=-\hat{K}_{1}{ }^{2}$ - $\hat{K}_{2}{ }^{2}+\hat{K}_{3}{ }^{2}=3 / 16$. The important observation here is that the log-radial self-adjoint generators in Eq. (53) are represented in quadratic functions (in exactly the same form as their Cartesian ones) of $\hat{p}_{r}, \hat{v}_{r}$, which themselves are self-adjoint in $\mathcal{L}_{2}^{(\eta)}\left(\mathbb{R}^{+}\right)$. However, their algebraic counterparts in the radial (nonlogarithmic) Hankel basis were also identified ${ }^{10}$ as generators of certain linear optical transformations induced by thin lenses, magnifiers, and free-space propagators [i.e., $\hat{J}_{i}(i=0,1,2)$ in Eqs. (26)-(30) in that reference]. However, unlike the case above, the linear canonical generators (let us denote them by $\hat{P}_{r}, \hat{r}$ ) on the half-line $\mathbb{R}^{+}$are not self-adjoint, nor do they have known extensions as such. This implies that these radial elements $\hat{P}_{r}, \hat{r}$ do not support unitary WH representations of the type shown in Eq. (42), which can be summarized in the following diagram:

$$
\begin{array}{cccc}
C_{x}^{(g)} \hat{D}_{x} & \Leftrightarrow & c_{x}^{(g)} \\
\Uparrow & & \mathbb{\Downarrow} & c_{x}^{(g)} c_{y}^{(g)} \\
& {\left[C_{x}^{(g)} \hat{D}_{x}\right]\left[C_{y}^{(g)} \hat{D}_{y}\right]} & \Leftrightarrow & \Uparrow \\
\text { no! } \hat{D}_{x} \hat{D}_{y} \neq \oplus_{m} & \hat{D}_{r}^{(m)} \otimes \hat{D}_{\theta}^{(m)} & (x, y) \stackrel{?}{\leftrightarrow}(r, \theta) & \text { yes } \\
\Downarrow & & \Downarrow \\
? & \Leftrightarrow & \oplus_{m} c_{r}^{(g)^{(m)}} \exp (\operatorname{im} \theta) .
\end{array}
$$

$$
\lim _{g_{i} \rightarrow 1} c_{r}^{\left(g_{i}\right)}\left(\lambda_{1}, \lambda_{2}\right)=\delta\left(\lambda_{1}-\lambda_{2}\right) .
$$

The log-radial coordinate representations can be found by calculation of the Mellin transform of Eq. (48). But there is an easier way. We continue to use the eigenrep-
On the left-hand side of correspondence (55) we have what is essentially the unitary representation shown in Eq. (42). On the corresponding right-hand side we have the integral operator representation shown in Eq. (48). On extension of the scheme to two or more Cartesian dimensions the correspondence is manifested, as expected, 
by a direct product in the WH basis and an ordinary product in the function space. The integral operator representations on the right can be written in terms of the helicity $(m)$ expansion of the wave field in the nonlogarithmic radial Hankel basis, as shown in Ref. 10. They are structurally different from the log-radial coordinate representation of those shown in Eqs. (49)-(51). The former (nonlogarithmic Hankel) ones do not have WH operator kernels [via correspondence (55)], whereas the log-radial representations of the WH kernels of Eqs. (49)(51) exist and are given by Eqs. (46).

From the optics point of view it is desirable to formulate a Wigner function covariant under the action of linear optical devices. For convenience, let us call the latter the linear optical covariance. This covariance arises in the $\operatorname{Sp}_{x}(2, \mathbb{R}) \otimes \operatorname{Sp}_{y}(2, \mathbb{R})$ subgroup decomposition of the group of LCT in two Cartesian dimensions. This subgroup further decomposes ${ }^{10}$ into an infinite helicity $(\mathrm{m})$ sum of the actions of $\operatorname{Sp}_{r}^{(m)}\left(2, \mathbb{R}^{+}\right)$, each acting irreducibly in the definite helicity $(m)$ subspace for integer $m$. Moregeneral ones for $\operatorname{Sp}(4, \mathbb{R})$ have also been reported. ${ }^{11}$ What the diagram in correspondence (55) then says is that, within a logarithmic or nonlogarithmic radial coordinate representation achieving linear optical covariance and canonicality simultaneously-in the context of WH representations-may be difficult.

However, in the log-radial representation, it is still possible to approximate the effective action of some optical elements in certain regions of the radial space by use of the combinations of the log-radial Galilean and the $\operatorname{Sp}(2, \mathbb{R})$ generators. The first example is $\exp \left(i \beta \hat{p}_{r}\right)$ as a dilation generator, whose effect is a magnification of the initial wave field as $\exp \left(i \ln s \hat{p}_{r}\right): \quad \psi(r) / \sqrt{r^{\eta-1 / 2}}$ $\rightarrow \sqrt{s} \psi(s r) / \sqrt{r^{\eta-1 / 2}}$ after one accounts for the appropriate weight factor in the denominators. The second example is the multiplication by a Gaussian phase, whose effect is generated by thin lenses. By direct inspection of Eq. (37) in the range $|1-r| \ll 1$ (remember that $r$ is in units of the optical wavelength $\chi$ ), we can observe that the local phase shift is effectively approximated by a Gaussian and is expressed in terms of the generators $\hat{K}_{1}, \hat{K}_{2}$ and $\hat{v}_{r}$ as

$$
\begin{aligned}
\exp \left[i \alpha\left(r^{2}-1\right)\right] \psi(r) \simeq & \left\{\exp \left[i 2 \alpha\left(\hat{v}_{r}+\hat{v}_{r}^{2}\right)\right]\right\} \psi(r) \\
= & \exp \left(i 2 \alpha \hat{v}_{r}\right) \\
& \times \exp \left[i 4 \alpha\left(\hat{K}_{1}-\hat{K}_{2}\right)\right] \psi(r)
\end{aligned}
$$

up to terms $\mathcal{O}\left[\exp \left(i \alpha \hat{v}_{r}^{3}\right)\right]$ on the right-hand side, provided that $|\ln r| \ll 1$.

(4) The inner product property reads as follows:

$$
\begin{aligned}
\int_{-\infty}^{\infty} \mathrm{d} v_{r} \int_{-\infty}^{\infty} \mathrm{d} p_{r} & W_{\psi}\left(p_{r}, v_{r}\right) W_{\phi}\left(p_{r}, v_{r}\right) \\
& =\frac{1}{2 \pi}\left|\int_{-\infty}^{\infty} \mathrm{d} v \exp (2 \eta v) \psi^{*}(\exp v) \phi(\exp v)\right|^{2} \\
& =\frac{1}{2 \pi}\left|(\psi, \phi)_{r}\right|^{2}
\end{aligned}
$$

Radial Wigner function in a noncovariant form. Our purpose in this section is to learn whether one can define a nonlinear canonical transformation ${ }^{12}$ from $W_{\psi}\left(p_{r}, v_{r}\right)$ to another Wigner function $\omega_{\psi}\left(P_{r}, r\right)$ on the basis of the more desirable canonical pair $P_{r}, r$ without being blocked by the nonexisting radial (nonlogarithmic) Weyl correspondence. One can partially achieve this by first devising a canonical transformation generator from $p_{r}, v_{r}$ $=\ln r$ to $P_{r}=\exp \left(-v_{r}\right) p_{r}, r=\exp \left(v_{r}\right)$.

Let us now consider the following Fourier-Mellin transform $\tilde{\psi}\left(v_{r}\right)$ of a radial signal $\psi(r)$ as

$$
\begin{aligned}
\widetilde{\psi}\left(v_{r}\right) & =\left(\mathcal{F}_{M}: \psi\right)\left(v_{r}\right) \\
& =\int_{-\infty}^{\infty} \frac{\mathrm{d} \lambda}{\sqrt{2 \pi}} \exp \left(-i \lambda v_{r}\right)\left(\varphi_{\lambda}, \psi\right)_{r} \\
& =\exp \left(\eta v_{r}\right) \psi\left(\exp v_{r}\right) .
\end{aligned}
$$

Equation (58) is a unitary transformation between functions in the radial $r$ representation and functions in the radial $v_{r}=\ln r$ representation. The impulse response ${ }^{13}$ corresponding to this coordinate transformation is

$$
\begin{aligned}
& g_{v_{r}}(r)=\exp \left(-\eta v_{r}\right) \delta\left(v_{r}-\ln r\right), \\
& \tilde{\psi}\left(v_{r}\right)=\left(g_{v_{r}}, \psi\right)_{r}=\exp \left(\eta v_{r}\right) \psi\left(\exp v_{r}\right) .
\end{aligned}
$$

Using Eqs. (59), we find that Eq. (30), as expected, adopts the standard form

$$
\begin{aligned}
W_{\psi}\left(p_{r}, v_{r}\right)= & \frac{1}{2 \pi} \int_{-\infty}^{\infty} \mathrm{d} \beta_{r} \exp \left(-i \beta_{r} p_{r}\right) \tilde{\psi}^{*}\left(v_{r}+\beta_{r} / 2\right) \\
& \times \tilde{\psi}\left(v_{r}-\beta_{r} / 2\right) .
\end{aligned}
$$

Consider a new pseudo Wigner function of the form

$$
\begin{array}{r}
\omega_{\psi}\left(P_{r}, r\right)=\frac{1}{2 \pi} \int_{\mathbb{R}^{+}} \mathrm{d} s s^{-i r P_{r}-1} r^{2 \eta} \psi^{*}(\sqrt{s} r) \psi(r / \sqrt{s}), \\
\psi(r) \in \mathcal{L}_{2}^{(\eta)}\left(\mathbb{R}^{+}\right) .
\end{array}
$$

Using Eqs. (58) and (59), we can relate Eqs. (60) and (61) through

$$
\begin{aligned}
W_{\psi}\left(p_{r}, v_{r}\right) & =\int \mathrm{d} P_{r} \mathrm{~d} r \mathcal{T}\left(p_{r}, v_{r} ; P_{r}, r\right) w_{\psi}\left(P_{r}, r\right), \\
\mathcal{T} & =\delta\left(r P_{r}-p_{r}\right) \delta\left(v_{r}-\ln r\right),
\end{aligned}
$$

which does correspond to a canonical transformation; i.e., $\left(p_{r}, v_{r}\right) \rightarrow\left[P_{r}=\exp \left(-v_{r}\right) p_{r}, r=\exp \left(v_{r}\right)\right]$. Some of the properties of the pseudo Wigner function read as follows:

(1) The pseudo Wigner function is real.

(2) Its normalization is given by $\int \mathrm{d} p_{r} \mathrm{~d} v_{r} W_{\psi}$ $=\int \mathrm{d} P_{r} \mathrm{~d} r \omega_{\psi}=1$. Essentially,

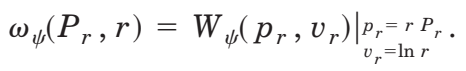

(3) The marginal probability for $r$ is obtained, as expected, as

$$
\int \mathrm{d} P_{r} \omega_{\psi}\left(P_{r}, r\right)=r^{2 \eta-1}|\psi(r)|^{2} .
$$

(4) Under scale changes induced by the operator $\exp \left(i \beta \hat{p}_{r}\right)$ in Eq. (35), one has 


$$
\psi \stackrel{\exp \left(i \beta \hat{p}_{r}\right)}{\longrightarrow} \psi^{\prime} \Rightarrow \omega_{\psi}\left(P_{r}, r\right)=\omega_{\psi^{\prime}}\left(\exp (\beta) P_{r}, \exp (-\beta) r\right)
$$

Hence the covariance is manifest under radial dilations. If one considers $\sqrt{r^{2 \eta-1}} \psi(r) \in \mathcal{L}_{2}^{(1 / 2)}\left(\mathbb{R}^{+}\right)$, one has the Hankel-type normalization used in Ref. 10. Expression (66), then, states the covariance of the pseudo Wigner function under the scaling generator $\hat{J}_{2}^{(m)}$ therein. By contrast, the pseudo Wigner function is not covariant under log-radial $\operatorname{Sp}(2, \mathbb{R})$ or under any local phase shift induced by the operator $\exp [i \alpha g(\hat{r})]$, where $g$ is any function. It is also not covariant under $\operatorname{SP}_{r}^{(m)}\left(2, \mathbb{R}^{+}\right)$other than under the scaling transformation. It is expected that the $\mathrm{Sp}(2, \mathbb{R})$ covariance would be lost through the transformation in Eqs. (62). The log-radial conjugate coordinates can mix under the action of the off-diagonal elements of the LCT because they have the same domain, which is simply R. The off-diagonal LCT's on the other radial pair $\left(P_{r}, r\right)$ are forbidden. This is because Eq. (62) implies that $P_{r} \in \mathbb{R}$ and that $r \in \mathbb{R}^{+}$. Hence the conjugate coordinates in the new pair cannot covariantly mix with each other. Indeed, the scaling generators $\hat{K}_{3}$ of the log-radial $\mathrm{Sp}(2, \mathbb{R})$ in Eqs. (53) and the scaling generator $\hat{J}_{2}^{(m)}$ in $\mathrm{Sp}_{r}^{(m)}\left(2, \mathrm{R}^{+}\right)$are related to each other by the same canonical transformation as in Eq. (62). They also have no offdiagonal elements. Hence they leave both Wigner functions covariant. Similarly, we will observe in Subsection 2.A.2, on the angular part, that the standard LCT covariance is absent in the angular Wigner function inasmuch as the domains of the angular and the angularmomentum variables are quite distinct from each other.

With regard to the fact that the $P_{r}$ distribution is represented by

$$
\int_{\mathrm{R}^{+}} \mathrm{d} r \omega_{\psi}\left(P_{r}, r\right)
$$

we can say only that it is real by construction of $\omega_{\psi}\left(P_{r}, r\right)$ and that it is normalized to unity. Beyond this trivial result, it should also be determined whether it is nonnegative for acceptability as a distribution.

\section{Angular Part}

The angular phase-space representations have been one of the long-standing problems since the 1920's because of their connection with one of the fundamental anomalies in quantum mechanics. ${ }^{14,15}$ The standard canonical coordinates with unbounded (continuous or discrete) spectra are not in the trace class, and their standard commutation rule violates a fundamental trace identity, which prevents a well-defined unitary phase operator to exist. ${ }^{15}$ The resolution of this problem requires a different starting point than the standard continuous phase space: a discrete and finite-dimensional phase space with periodic boundaries, which is effectively a discrete torus. ${ }^{16}$ One then defines the standard quantum-mechanical phase space in a semidiscrete limit ${ }^{17}$ in which one increases the number of discrete points in both directions in the phase space to infinity in such a way that one of the discrete coordinates approaches a continuous and bounded phase variable, $-\pi \leqslant v_{\theta}<\pi$, and the other one remains discrete as its conjugate partner (the angular momentum), $p_{\theta} \in \mathbb{Z}$. The geometry of the semidiscrete limit is visualized as a cylinder of rings of unit radius. Each ring is separated from the other by a unit of angular momentum, with the rings corresponding to the boundaries of the cylinder along the axis located at $\pm \infty$. A point in the phase space is then defined by an angular variable (the phase $v_{\theta}$ ) parameterizing the ring and by a discrete number (the angular momentum or the helicity factor, $-\infty<p_{\theta}<\infty$ ) parameterizing which ring, along the axis, that it is referred to.

The rigorous definition of the angular Wigner function requires this specific limiting procedure from a fully discrete to a semidiscrete form, as described above. For clarity here we will start from the semidiscrete formalism and refer to Refs. 16 and 17 for details.

The semidiscrete angular kernel, as the angular analog of Eqs. (14), basically amounts to construction of a semidiscrete WH operator basis $\hat{D}_{\theta}(n, \zeta)$, with $n \in \mathbb{Z}$ and $\zeta$ $\in[-\pi, \pi)$, whose action on functions $F(\phi)$ on the unit circle is defined by

$$
\begin{array}{r}
\hat{D}_{\theta}(n, \zeta) F(\phi) \equiv \exp (\operatorname{in} \zeta / 2) \exp (\operatorname{in} \phi) F(\phi+\zeta), \\
-\pi \leqslant \phi<\pi .
\end{array}
$$

For construction of the angular Wigner function, it will also be necessary to know the action of $\hat{D}_{\theta}(n, \zeta)$ on the Fourier transform of $F$. This Fourier transform is defined by

$$
f_{m}=\frac{1}{\sqrt{2 \pi}} \int_{-\infty}^{\infty} \mathrm{d} \phi F(\phi) \exp (-i m \phi), \quad-\infty<m<\infty,
$$

where $m$ must have the same domain as does $n$ in relations (67). For $f_{m}$, we find that

$$
\hat{D}_{\theta}(n, \zeta) f_{m}=\exp (-i n \zeta) \exp (i m \zeta) f_{m-n} .
$$

It can be seen that, if $F$ and $G$ are two functions on the unit circle, $\hat{D}_{\theta}(n, \zeta)$ is unitary,

$$
\begin{aligned}
\left\langle F, \hat{D}_{\theta}(n, \zeta) G\right\rangle_{\theta} & =\left\langle\hat{D}_{\theta}^{\dagger}(n, \zeta) F, G\right\rangle_{\theta} \\
& =\left\langle\hat{D}_{\theta}{ }^{-1}(n, \zeta) F, G\right\rangle_{\theta},
\end{aligned}
$$

over the inner product in the angle representation

$$
\langle F, G\rangle_{\theta}=\int_{-\pi}^{\pi} \mathrm{d} \phi F^{*}(\phi) G(\phi)
$$

or in the discrete angular-momentum representation

$$
\langle F, G\rangle_{\theta}=\sum_{m=-\infty}^{\infty} f_{m}^{*} g_{m}
$$

and it satisfies

$$
\begin{aligned}
\hat{D}_{\theta}(0,0) & =\hat{\mathbb{I}} \\
\hat{D}_{\theta}^{\dagger}(n, \zeta) & =\hat{D}_{\theta}^{-1}(n, \zeta)=\hat{D}_{\theta}(-n,-\zeta) \\
\operatorname{Tr}\left[\hat{D}_{\theta}(n, \zeta)\right]= & 2 \pi \delta(\zeta) \delta_{n, 0}, \\
\hat{D}_{\theta}(n, \zeta) \hat{D}_{\theta}\left(n^{\prime}, \zeta^{\prime}\right)= & \exp \left[-i\left(n \zeta^{\prime}-\zeta n^{\prime}\right) / 2\right] \\
& \times \hat{D}_{\theta}\left(n+n^{\prime}, \zeta+\zeta^{\prime}\right)
\end{aligned}
$$


Equation (75), where Tr stands for the trace of the matrix elements of $\hat{D}_{\theta}(n, \zeta)$, guarantees that the angular WH basis is orthogonal. To calculate Eq. (75) we consider the simplest complete and orthonormal basis functions on the unit circle as the Fourier basis $L_{m}(\phi)$ by which the trace is defined. This definition is written as

$$
\begin{aligned}
\operatorname{Tr}\left[\hat{D}_{\theta}(n, \zeta)\right] & \equiv \sum_{m=-\infty}^{\infty}\left\langle L_{m}, \hat{D}_{\theta}(n, \zeta) L_{m}\right\rangle_{\theta}, \\
L_{m}(\phi) & =\frac{1}{\sqrt{2 \pi}} \exp (i m \phi) .
\end{aligned}
$$

By use of Eqs. (71) and (77) one can derive Eq. (75). The construction of the angular kernel follows by direct analogy with Eqs. (14). We introduce the angular kernel $\hat{\Delta}_{\theta}\left(p_{\theta}, v_{\theta}\right)$ as

$$
\begin{aligned}
\hat{\Delta}_{\theta}\left(p_{\theta}, v_{\theta}\right)= & \frac{1}{2 \pi} \sum_{n=-\infty}^{\infty} \int_{-\pi}^{\pi} \frac{\mathrm{d} \zeta}{2 \pi} \\
& \times \exp \left[-i\left(n v_{\theta}+\zeta p_{\theta}\right)\right] \hat{D}_{\theta}(n, \zeta),
\end{aligned}
$$

where $-\pi \leqslant v_{\theta}<\pi$ and $p_{\theta} \in \mathbb{Z}$. These properties of $\hat{D}_{\theta}$ translate to those of the angular kernel $\hat{\Delta}_{\theta}\left(p_{\theta}, v_{\theta}\right)$ as

$$
\begin{aligned}
\hat{\Delta}_{\theta}\left(p_{\theta}, v_{\theta}\right) & =\hat{\Delta}_{\theta}^{\dagger}\left(p_{\theta}, v_{\theta}\right), \\
\operatorname{Tr}\left\{\hat{\Delta}_{\theta}\left(p_{\theta}, v_{\theta}\right)\right\} & =\frac{1}{2 \pi} \\
\operatorname{Tr}\left\{\hat{\Delta}_{\theta}\left(p_{\theta}, v_{\theta}\right) \hat{\Delta}_{\theta}\left(p_{\theta}^{\prime}, v_{\theta}^{\prime}\right)\right\} & =\frac{1}{2 \pi} \delta_{p_{\theta}, p_{\theta}^{\prime}} \delta\left(v_{\theta}-v_{\theta}^{\prime}\right), \\
\int_{-\pi}^{\pi} \mathrm{d} v_{\theta} \sum_{p_{\theta}=-\infty}^{\infty} \hat{\Delta}_{\theta}\left(p_{\theta}, v_{\theta}\right) & =\hat{\mathbb{I}}, \\
\int_{-\pi}^{\pi} \mathrm{d} v_{\theta} \hat{\Delta}_{\theta}\left(p_{\theta}, v_{\theta}\right) & =\hat{\widetilde{P}}_{\theta}\left(p_{\theta}\right), \\
\sum_{p_{\theta}=-\infty}^{\infty} \hat{\Delta}_{\theta}\left(p_{\theta}, v_{\theta}\right) & =\hat{\mathcal{P}}_{\theta}\left(v_{\theta}\right),
\end{aligned}
$$

where the angular projection operators $\hat{\mathcal{P}}_{\theta}\left(v_{\theta}\right)$ and $\widetilde{\widetilde{\mathcal{P}}}_{\theta}\left(p_{\theta}\right)$ are defined in a manner similar to that of the radial ones in Eqs. (26) and (28) as

$$
\begin{aligned}
& \hat{\mathcal{P}}_{\theta}\left(v_{\theta}\right) \hat{\mathcal{P}}_{\theta}\left(v_{\theta}^{\prime}\right)=\delta\left(v_{\theta}-v_{\theta}^{\prime}\right) \hat{\mathcal{P}}_{\theta}\left(v_{\theta}\right), \quad \int \mathrm{d} v_{\theta} \hat{\mathcal{P}}\left(v_{\theta}\right)=\hat{\mathrm{I}}, \\
& \hat{\widetilde{\mathcal{P}}}_{\theta}\left(p_{\theta}\right) \widetilde{\widetilde{\mathcal{P}}}_{\theta}\left(p_{\theta}^{\prime}\right)=\delta_{p_{\theta}, p_{\theta}^{\prime}} \hat{\widetilde{\mathcal{P}}}_{\theta}\left(p_{\theta}\right), \quad \sum_{p_{\theta}=-\infty}^{\infty} \widehat{\widetilde{\mathcal{P}}}_{\theta}\left(p_{\theta}\right)=\hat{\mathrm{I}} \\
& \left\langle F, \hat{\mathcal{P}}_{\theta}\left(v_{\theta}\right) G\right\rangle_{\theta} \equiv F^{*}\left(v_{\theta}\right) G\left(v_{\theta}\right), \\
& \left\langle F, \hat{\widetilde{\mathcal{P}}}_{\theta}\left(p_{\theta}\right) G\right\rangle_{\theta} \equiv f_{p_{\theta}}^{*} g_{p_{\theta}} .
\end{aligned}
$$

Angular Wigner Function and Its Properties. The representation-independent form of the angular Wigner function can be defined as

$$
W_{F}\left(p_{\theta}, v_{\theta}\right)=\left\langle F, \hat{\Delta}_{\theta}\left(p_{\theta}, v_{\theta}\right) F\right\rangle_{\theta},
$$

which is represented in the angular coordinate basis as

$$
\begin{aligned}
W_{F}\left(p_{\theta}, v_{\theta}\right)= & \frac{1}{2 \pi} \int_{-\pi}^{\pi} \mathrm{d} \zeta \exp \left(-i \zeta p_{\theta}\right) F^{*}\left(v_{\theta}-\zeta / 2\right) \\
& \times F\left(v_{\theta}+\zeta / 2\right)
\end{aligned}
$$

The angular-momentum representation of Eq. (88) requires the use of a fractionally shifted angularmomentum spectrum. ${ }^{16,17}$ In our discussions here we shall construct the Wigner function in the angular coordinate representations to avoid this sort of abstraction. The static properties of the angular Wigner function follow directly from Eq. (89). These are as follows:

(1) $W_{F}\left(p_{\theta}, v_{\theta}\right)$ is real, namely,

$$
W_{F}\left(p_{\theta}, v_{\theta}\right)=W_{F} *\left(p_{\theta}, v_{\theta}\right)
$$

which follows directly from Eq. (79).

(2) The integral (sum) of $W_{F}\left(p_{\theta}, v_{\theta}\right)$ with respect to one of the phase-space variables $p_{\theta}, v_{\theta}$ yields the marginal probability with respect to the other variable:

$$
\begin{aligned}
& \int_{-\pi}^{\pi} \mathrm{d} v_{\theta} W_{F}\left(p_{\theta}, v_{\theta}\right)=\left|f_{p_{\theta}}\right|^{2}, \\
& \sum_{p_{\theta}=-\infty}^{\infty} W_{F}\left(p_{\theta}, v_{\theta}\right)=\left|F\left(v_{\theta}\right)\right|^{2},
\end{aligned}
$$

which follow directly from Eqs. (79) and (80).

(3) Static covariance properties: Unlike the radial part, the angular Wigner function is not covariant under the action of the LCT's. This is because $v_{\theta}$, with a finite and continuous support \{i.e., $\left.v_{\theta} \in[-\pi, \pi)\right\}$ and $p_{\theta}$, with an infinite and discrete one (i.e., $p_{\theta} \in Z$ ), do not mix. For this reason, below we consider only the Galilean transformations for the angular part.

Angular Galilean transformations. These are performed as follows.

(a) Define a new function $F^{\prime}(\phi)$ as

$$
F(\phi)=\exp \left(i \zeta^{\prime} \hat{p}_{\theta}\right) F^{\prime}(\phi)=F^{\prime}\left(\phi+\zeta^{\prime}\right), \quad \zeta^{\prime} \in \mathbb{R} .
$$

Inserting Eq. (93) into Eqs. (88) and (89), we find that

$$
W_{F}\left(p_{\theta}, v_{\theta}\right)=W_{F^{\prime}}\left(p_{\theta}, v_{\theta}+\zeta^{\prime}\right) .
$$

(b) We now define the new function $F^{\prime}(\phi)$ as

$$
F(\phi)=\exp (i l \hat{\theta}) F^{\prime}(\phi)=\exp (i l \theta) F^{\prime}(\phi), \quad l \in \mathbb{Z} .
$$

Inserting Eq. (95) into Eqs. (88) and (89), we find that

$$
W_{F}\left(p_{\theta}, v_{\theta}\right)=W_{F^{\prime}}\left(p_{\theta}-l, v_{\theta}\right)
$$

Equations (94) and (96) describe the covariance of the angular Wigner function under Galilean transformations in the angular coordinate space.

(4) The inner product property reads as follows: 


$$
\begin{aligned}
\int_{-\pi}^{\pi} \mathrm{d} v_{\theta} & \sum_{p_{\theta}=-\infty}^{\infty} W_{F}\left(p_{\theta}, v_{\theta}\right) W_{G}\left(p_{\theta}, v_{\theta}\right) \\
\quad= & \frac{1}{2 \pi}\left|\int_{-\pi}^{\pi} \mathrm{d} v F^{*}(v) G(v)\right|^{2}=\frac{1}{2 \pi}\left|\langle F, G\rangle_{\theta}\right|^{2} .
\end{aligned}
$$

\section{B. Polar Representation of the Wigner Function}

We now demand that the $2 \mathrm{D}$ kernel $\hat{\Delta}(\mathbf{p}, \mathbf{q})$ in the Cartesian representation be equivalent to

$$
\hat{\Delta}(\mathbf{p}, \mathbf{q})=\hat{\Delta}_{r}\left(p_{r}, v_{r}\right) \otimes \hat{\Delta}_{\theta}\left(p_{\theta}, v_{\theta}\right)
$$

in the polar representation. We use the log-radial representation for the radial part in Eq. (98). It is clear that the radial and the angular kernels in Eq. (98), as well as their arguments $\left(p_{r}, v_{r}\right)$ and $\left(p_{\theta}, v_{\theta}\right)$, respectively, are independent of each other. The radial representation of the $2 \mathrm{D}$ Wigner function is then given by

$$
W_{\Psi}\left(p_{r}, v_{r} ; p_{\theta}, v_{\theta}\right)=\left\langle\Psi, \hat{\Delta}_{r}\left(p_{r}, v_{r}\right) \otimes \hat{\Delta}_{\theta}\left(p_{\theta}, v_{\theta}\right) \Psi\right\rangle_{r, \theta},
$$

where $\hat{\Delta}_{r}$ and $\hat{\Delta}_{\theta}$ independently act on the radial and the angular parts, respectively, of the wave function in Eq. (1). Using Eq. (1) in Eq. (99), we find that

$$
\begin{aligned}
W_{\Psi}\left(p_{r}, v_{r} ; p_{\theta}, v_{\theta}\right)= & 2 \pi \sum_{n, m \in Z}\left\langle L_{n}, \hat{\Delta}_{\theta}\left(p_{\theta}, v_{\theta}\right) L_{m}\right\rangle_{\theta} \\
& \times\left\langle\widetilde{\Psi}_{n}, \hat{\Delta}_{r}\left(p_{r}, v_{r}\right) \widetilde{\Psi}_{m}\right\rangle_{r}, \quad(100)
\end{aligned}
$$

where $\widetilde{\Psi}_{n}$ represents the radial part of the wave function $\Psi$ and $L_{n}$ is the Fourier basis, as given by Eqs. (77). The radial and the angular inner products $(,)_{r}$ and $(,)_{\theta}$ in Eq. (100) are defined in Eqs. (8) and (70), respectively. Performing the calculations in the angular part, we can present Eq. (100) in a more explicit form as

$$
\begin{aligned}
W_{\Psi}\left(p_{r}, v_{r} ; p_{\theta}, v_{\theta}\right) & \\
= & \frac{1}{2 \pi} \sum_{n, m \in Z} \exp \left[-i v_{\theta}(n-m)\right] \\
& \times\left(\int_{-\pi}^{\pi} \frac{\mathrm{d} \zeta}{2 \pi} \exp \left\{-i \zeta\left[p_{\theta}-(n+m) / 2\right]\right\}\right) \\
& \times\left\langle\widetilde{\Psi}_{n}, \hat{\Delta}_{r}\left(p_{r}, v_{r}\right) \widetilde{\Psi}_{m}\right\rangle_{r} .
\end{aligned}
$$

We now shift our attention to the radial part in Eq. (100). An arbitrary wave function $\Psi(\mathbf{r})$ in $\mathcal{L}_{2}(\mathbb{R})$ can be expanded in the polar representation $(r, \phi)$ of $\mathbf{r}$, as in Eq. (1). We define an inner product in this space as

$$
\langle\Psi, \Phi\rangle_{r, \theta}=\int_{\mathbb{R}^{d}} \mathrm{~d} \mathbf{r} \Psi^{*}(\mathbf{r}) \Phi(\mathbf{r}) .
$$

Comparing the radial part of Eq. (102) with the radial inner product given in Eq. (8), we find that $\eta=d / 2$, where $d$ is the dimension of the space. Here we are interested in $d=2$ only; hence $\eta=1$.

Here the most natural representation of the radial part is the Mellin basis $\varphi_{\lambda}(r)$, given in Eq. (7), in which we expand $\widetilde{\Psi}_{m}(r)$ as

$$
\tilde{\Psi}_{m}(r)=\int_{-\infty}^{\infty} \mathrm{d} \lambda A_{m}(\lambda) \varphi_{\lambda}(r), \quad A_{n}(\lambda)=\left\langle\varphi_{\lambda}, \tilde{\Psi}_{n}\right\rangle_{r}
$$

where we have used the orthogonality relations (9) and (10). From Eqs. (103) the radial part in Eq. (100) becomes

$$
\begin{aligned}
\left\langle\tilde{\Psi}_{n}, \hat{\Delta}_{r}\left(p_{r}, v_{r}\right) \tilde{\Psi}_{m}\right\rangle_{r}= & \int_{-\infty}^{\infty} \mathrm{d} \lambda A_{n}^{*}(\lambda) \int_{-\infty}^{\infty} \mathrm{d} \lambda^{\prime} A_{m}\left(\lambda^{\prime}\right) \\
& \times\left\langle\varphi_{\lambda}, \hat{\Delta}_{r}\left(p_{r}, v_{r}\right) \varphi_{\lambda^{\prime}}\right\rangle_{r} .
\end{aligned}
$$

The radial part in Eq. (104) is given in the radial coordinate representation by

$$
\begin{aligned}
\left\langle\varphi_{\lambda}, \hat{\Delta}_{r}\left(p_{r}, v_{r}\right) \varphi_{\lambda^{\prime}}\right\rangle_{r} \\
=\frac{1}{2 \pi} \int_{-\infty}^{\infty} \mathrm{d} \beta_{r} \exp \left(-i \beta_{r} p_{r}\right) \\
\quad \times \exp \left(n v_{r}\right) \varphi_{\lambda} *\left(v_{r}-\beta_{r} / 2\right) \varphi \lambda^{\prime}\left(v_{r}+\beta_{r} / 2\right) \\
=\frac{1}{2 \pi} \exp \left[-i v_{r}\left(\lambda-\lambda^{\prime}\right)\right] \delta\left(p_{r}-\frac{\lambda+\lambda^{\prime}}{2}\right) .
\end{aligned}
$$

Inserting Eq. (105) into Eq. (104), we find that

$$
\begin{aligned}
& \left\langle\tilde{\Psi}_{n}, \hat{\Delta}_{r}\left(p_{r}, v_{r}\right) \tilde{\Psi}_{m}\right\rangle_{r} \\
& \quad=\frac{1}{2 \pi} \int_{-\infty}^{\infty} \mathrm{d} \lambda \exp \left(-i \lambda v_{r}\right) A_{n} *\left(p_{r}+\lambda / 2\right) A_{m}\left(p_{r}-\lambda / 2\right),
\end{aligned}
$$

which we use in Eq. (101). Finally, an explicit form can be given by

$$
\begin{aligned}
W_{\Psi}\left(p_{r}, v_{r} ; p_{\theta}, v_{\theta}\right) \\
=\frac{1}{(2 \pi)^{3}} \sum_{n, m \in Z} \exp \left[-i v_{\theta}(n-m)\right] \\
\quad \times\left(\int_{-\pi}^{\pi} \mathrm{d} \zeta \exp \left\{-i \zeta\left[p_{\theta}-(n+m) / 2\right]\right\}\right) \\
\quad \times \int_{-\infty}^{\infty} \mathrm{d} \lambda \exp \left(-i \lambda v_{r}\right) A_{n} *\left(p_{r}+\lambda / 2\right) A_{m}\left(p_{r}-\lambda / 2\right) .
\end{aligned}
$$

\section{APPLICATION}

Although some very specific results exist, an explicitly canonical formulation of the polar (hence radial) Wigner function has not, to the author's knowledge, previously been tackled. The study that has most closely approached this goal is the recent work of Bastiaans and van de Mortel, ${ }^{18}$ whose research on the Wigner function of a circular aperture was based on an approximate Cartesian method specific to the model that they used. However, it has been shown here that it is possible to construct a generalized Wigner function formalism directly, starting from the radial (log or nonlog) coordinates for wave functions, which can be represented in a polar expansion of the form given in Eq. (1). Although it has been shown here that a Weyl correspondence for this 
transformation, relating the right-hand sides of Eqs. (2) and (98), may not exist, the question whether a coordinate transformer can be found in the phase space within the general context of Eq. (62) is very relevant from both the linear optics and the quantum mechanics points of view. The advantage of the Cartesian method, if it can be handled exactly and with sufficient generality, over the radial one is that the transformation under the action of linear optical systems coincides with the covariance transformations of the Wigner function. This is not the case in the direct radial (logarithmic or nonlogarithmic) situation, as we have already seen. In contrast, the advantage of the radial Wigner function is that it becomes favorable if the initial wave field is more appropriately represented in the angular-momentum $(m)$ expansion, as in Eq. (1). If the action of a linear optical device is represented by the function $\rho(r)$ multiplying the radial field $\psi(r)$, the corresponding Wigner function goes through a convolution similar to that of the Cartesian one, ${ }^{18}$ which can be written in one of the four equivalent ways between radial and angular coordinates and momenta as

$$
\begin{aligned}
W_{\psi^{\prime}}\left(p_{r}^{\prime}, v_{r}^{\prime} ; p_{\theta}, v_{\theta}^{\prime}\right)= & \int \mathrm{d} p_{r} \sum_{p_{\theta}} W_{\rho}\left(p_{r}^{\prime}-p_{r}, v_{r}^{\prime} ; p_{\theta}^{\prime}\right. \\
& \left.-p_{\theta}, v_{\theta}^{\prime}\right) W_{\psi}\left(p_{r}, v_{r}^{\prime} ; p_{\theta}, v_{\theta}^{\prime}\right) \\
= & \int \mathrm{d} v_{r} \sum_{p_{\theta}} W_{\rho}\left(p_{r}^{\prime}, v_{r}^{\prime}-v_{r} ; p_{\theta}^{\prime}\right. \\
& \left.-p_{\theta}, v_{\theta}^{\prime}\right) W_{\psi}\left(p_{r}^{\prime}, v_{r} ; p_{\theta}, v_{\theta}^{\prime}\right) \\
= & \ldots, \text { etc. }
\end{aligned}
$$

Below we calculate the Wigner function of the circular aperture, using the polar formalism developed here. We describe the wave function $\rho^{(\mathrm{CA})}(\mathbf{r})$ of the circular aperture as ${ }^{18}$

$$
\begin{aligned}
\rho^{(\mathrm{CA})}(\mathbf{r}) & =\frac{1}{\sqrt{\pi a^{2}}} \Theta(r-a) \\
& =\left\{\begin{array}{ll}
1 / \sqrt{\pi a^{2}} & \text { if } r \leqslant a \\
0 & \text { elsewhere }
\end{array} \Rightarrow \widetilde{\rho}_{m}^{(\mathrm{CA})}(r)\right. \\
& =\frac{\sqrt{2}}{a} \Theta(r-a) \delta_{m, 0}
\end{aligned}
$$

where we have used Eq. (1). Inserting relation (109) into Eq. (101), we find that

$$
W_{\rho^{(\mathrm{CA})}}\left(p_{r}, v_{r} ; p_{\theta}, v_{\theta}\right)=\frac{1}{2 \pi} \delta_{p \theta, 0} W_{\tilde{\rho}^{(\mathrm{CA})}}\left(p_{r}, v_{r}\right) .
$$

Using Eq. (30), we calculate the radial part in Eq. (110) from

$$
\begin{aligned}
W_{\tilde{\rho}^{(\mathrm{CA})}}\left(p_{r}, v_{r}\right) \\
=\frac{1}{\pi a^{2}} \int_{-\infty}^{\infty} \mathrm{d} \beta_{r} \exp \left(-i \beta_{r} p_{r}\right) \exp \left(2 v_{r}\right) \\
\quad \times \Theta\left[a-\exp \left(v_{r}-\beta_{r} / 2\right)\right] \Theta\left[a-\exp \left(v_{r}+\beta_{r} / 2\right)\right],
\end{aligned}
$$

where we have used $\eta=1$. Clearly, the radial Wigner function above vanishes if $\ln a \leqslant v_{r}$. A simple calculation yields

$$
\begin{aligned}
& W_{\rho^{(\mathrm{CA})}(}\left(p_{r}, v_{r} ; p_{\theta}, v_{\theta}\right) \\
& =\frac{1}{\pi^{2} a^{2}} \delta_{p_{\theta}, 0} \exp \left(2 v_{r}\right) \frac{1}{p_{r}} \\
& \quad \times \sin \left[2 p_{r}\left(\ln a-v_{r}\right)\right] \quad \text { if } v_{r} \leqslant \ln a
\end{aligned}
$$

and zero elsewhere. Equation (112) is depicted in Fig. 1 for the unit aperture radius $a=1$.

One can obtain the marginal probability distributions for the phase-space variables by integrating (summing) all other variables as

$$
\begin{aligned}
\mathcal{D}_{r}^{(\mathrm{CA})}\left(v_{r}\right) & =\sum_{p_{\theta}=-\infty}^{\infty} \int_{-\pi}^{\pi} \mathrm{d} v_{\theta} \int_{-\infty}^{\infty} \mathrm{d} p_{r} W_{\rho}(\mathrm{CA})\left(p_{r}, v_{r} ; p_{\theta}, v_{\theta}\right) \\
& =\frac{2}{a^{2}} \exp \left(2 v_{r}\right) \Theta\left[a-\exp \left(v_{r}\right)\right], \\
\mathcal{D}_{r}^{(\mathrm{CA})}\left(p_{r}\right) & =\sum_{p_{\theta}=-\infty}^{\infty} \int_{-\pi}^{\pi} \mathrm{d} v_{\theta} \int_{-\infty}^{\infty} \mathrm{d} v_{r} W_{\rho}(\mathrm{CA})\left(p_{r}, v_{r} ; p_{\theta}, v_{\theta}\right) \\
& =\frac{1}{\pi} \frac{1}{1+p_{r}{ }^{2}}, \\
\mathcal{D}_{\theta}^{(\mathrm{CA})}\left(v_{\theta}\right) & =\sum_{p_{\theta}=-\infty}^{\infty} \int_{-\infty}^{\infty} \mathrm{d} v_{r} \int_{-\infty}^{\infty} \mathrm{d} p_{r} W_{\rho} \mathrm{CA}\left(p_{r}, v_{r} ; p_{\theta}, v_{\theta}\right) \\
& =\frac{1}{2 \pi}, \\
\mathcal{D}_{\theta}^{(\mathrm{CA})}\left(p_{\theta}\right) & =\int_{-\pi}^{\pi} \mathrm{d} v_{\theta} \int_{-\infty}^{\infty} \mathrm{d} v_{r} \int_{-\infty}^{\infty} \mathrm{d} p_{r} W_{\rho^{(}(\mathrm{CA})}\left(p_{r}, v_{r} ; p_{\theta}, v_{\theta}\right) \\
& =\delta_{p_{\theta}, 0},
\end{aligned}
$$

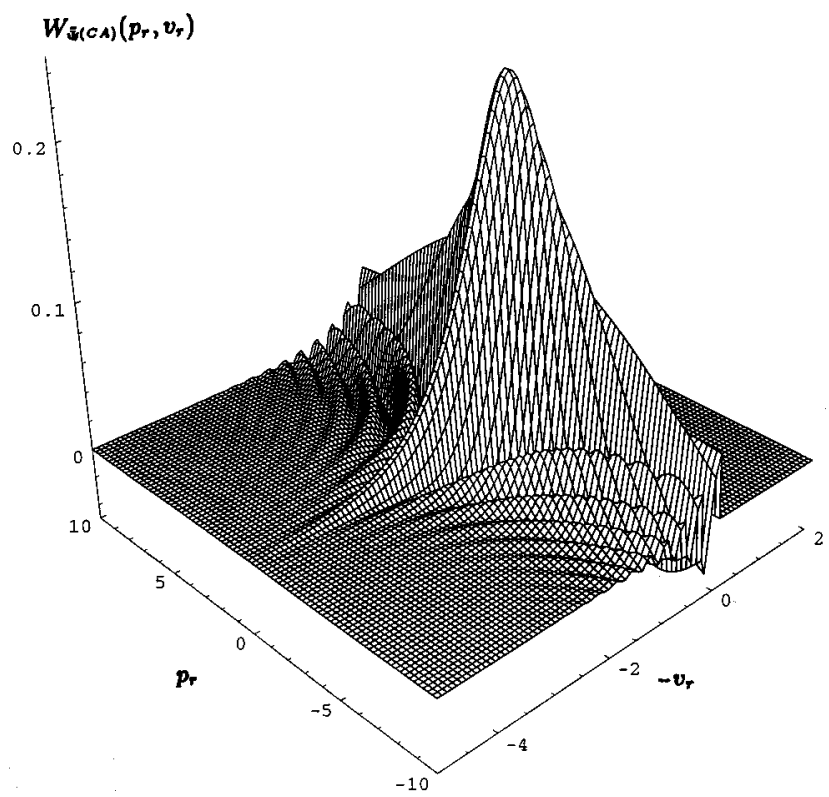

Fig. 1. Radial part of the Wigner function $\left[W_{\tilde{\Psi}^{(\mathrm{CA})}}\left(p_{r}, v_{r}\right)\right.$ in relations (112)] for the circular aperture of unit radius versus the phase-space variables $p_{r}, v_{r}$. The Wigner function vanishes for $v_{r} \leqslant 0$. 
where all marginal distributions are normalized to unity. One can also equivalently represent Eq. (111) in terms of the pseudo Wigner function given in Eq. (61), using Eq. (63).

\section{CONCLUSIONS}

Formulation of physical systems in the phase space by use of Wigner functions has become a powerful tool in the application of the fundamental phase-space concepts, ${ }^{5}$ particularly to signal processing ${ }^{19}$ and to classical ${ }^{18,20}$ as well as quantum optics. ${ }^{21}$ The existence of a complete orthogonal and unitary Weyl-Heisenberg operator basis, given, for example, by the expressions presented in Eqs. (4), for the Cartesian basis, or by those presented in Eqs. (14), for the radial one, is the crucial element for the Wigner function formulation of the phase space.

It is desirable to adopt the symmetries of the physical system in its representations on the phase space. Perhaps the action-angle basis, as built on the idea of representing a physical system by its maximum number of symmetry generators, does this in the most natural way. ${ }^{16,17}$ The polar canonical phase-space representations adopted in this study are expected to be important for paraxial optical systems as well as other systems in which a rotational symmetry around a particular axis is present. A simple example from classical electromagnetism is presented in Section 3.

Other immediate areas of application of the polar Wigner function are expected to be in the field of atomic and condensed-matter physics. Specifically, studies on quantum wires and dots, as well as studies on BoseEinstein phase-space condensation of atomic systems under external potentials with certain rotational symmetry properties, can be facilitated by use of the polar Wigner function formalism.

\section{ACKNOWLEDGMENTS}

The author is particularly grateful to K. B. Wolf (Centro Internacional de Ciencias/Cuernavaca, Mexico), for providing a copy of Ref. 10 prior to its publication and for stimulating conversations, and to $\mathrm{H}$. Özaktaş (Bilkent University), for informing the author about Ref. 18. Discussions with L. Barker (Bilkent University), A. Verçin (Ankara University), and C. Zachos at Argonne National Laboratories, where parts of this manuscript were written, are also gratefully acknowledged. This work was supported in part by the U.S. Department of Energy, Division of High Energy Physics, under contract W-31-109Eng-38.

T. Hakioğlu can be reached by e-mail at hakioglu @theory.hep.anl.gov.

\section{REFERENCES}

1. H. Weyl, "Quantenmechanik und Gruppentheorie," Z. Phys. 46, 1-39 (1927).

2. E. P. Wigner, "On the quantum corrections for thermodynamic equilibrium," Phys. Rev. 40, 749-760 (1932).

3. H. J. Groenewold, "On the principles of elementary quan- tum mechanics," Physica (Amsterdam) 12, 405-460 (1946); J. E. Moyal, "Quantum mechanics as a statistical theory," Proc. Cambridge Philos. Soc. 45, 99-124 (1949).

4. A. Verçin, "Metaplectic covariance of the Weyl-WignerGroenewold-Moyal quantization and beyond," Ann. Phys. (N.Y.) 266, 503-523 (1998); T. Dereli and A. Verçin, " $W_{\infty}$ covariance of the Weyl-Wigner-Groenewold-Moyal quantization," J. Math. Phys. 38, 5515-5530 (1997).

5. M. Hillery, R. F. O'Connell, M. O. Scully, and E. P. Wigner, "Distribution functions in physics: fundamentals," Phys. Rep. 106, 121-167 (1984); N. L. Balazs and B. K. Jennings, "Wigner functions and other distribution functions in mock phase spaces," Phys. Rep. 104, 347-391 (1984); R. G. Littlejohn, "Semiclassical evolution of wave packets," Phys. Rep. 138, 193-291 (1986).

6. P. A. M. Dirac, The Principles of Quantum Mechanics (Clarendon, Oxford, 1958).

7. J. Twamley, "Quantum distribution functions for radial observables,” J. Phys. A 31, 4811-4819 (1998).

8. A. Erdelyi, W. Magnus, F. Oberhettinger, and F. G. Tricomi, eds., Tables of Integral Transforms (McGraw-Hill, New York, 1954), Vols. 1 and 2.

9. M. Moshinsky and C. Quesne, "Linear canonical transformations and their unitary representations," J. Math. Phys. 12, 1772-1780 (1971); "Canonical transformations and matrix elements," 1780-1784 (1971); K. B. Wolf, Integral Transforms in Science and Engineering (Plenum, New York, 1979).

10. N. M. Atakishiyev, S. M. Nagiyev, L. E. Vicent, and K. B. Wolf, "Covariant discretization of axis-symmetric linear optical systems," J. Opt. Soc. Am. A 17, 2301-2314 (2000).

11. R. Simon and K. B. Wolf, "Structure of the set of paraxial optical systems," J. Opt. Soc. Am. A 17, 342-355 (2000).

12. T. Curtright, D. Fairlie, and C. Zachos, "Features of timeindependent Wigner functions," Phys. Rev. D 58, 025002-1025002-14 (1998).

13. O. Bryngdahl, "Geometrical transforms in optics," J. Opt. Soc. Am. 64, 1092-1099 (1974); M. J. Bastiaans, "The Wigner distribution function applied to optical signals and systems," Opt. Commun. 25, 26-30 (1978).

14. P. A. M. Dirac, "The quantum theory of the emission and absorption of radiation," Proc. R. Soc. London Ser. A 114, 243-265 (1927).

15. K. Fujikawa, L. C. Kwek, and C. H. Oh, " $q$-deformed oscillator algebra and an index theorem for the photon phase operator," Mod. Phys. Lett. A 10, 2543-2551 (1995); "A Schwinger term in $q$-deformed su(2) algebra," 12, 403-409 (1997).

16. T. Hakioğlu, "Finite dimensional Schwinger basis, deformed symmetries, Wigner function and an algebraic approach to quantum phase," J. Phys. A 31, 6975-6994 (1998); "Linear canonical transformations and the quantum phase: unified canonical and algebraic approach," 32, 4111-4130 (1999); T. Hakioğlu and K. B. Wolf, "The canonical Kravchuk basis for discrete quantum mechanics," J. Phys. A 33, 3313-3324 (2000).

17. T. Hakioğlu and E. Tepedelenlioğlu, "The action-angle Wigner function: a discrete and algebraic phase space formalism," J. Phys. A 33, 6357-6383 (2000).

18. M. J. Bastiaans and P. G. J. van de Mortel, "Wigner distribution function of a circular aperture," J. Opt. Soc. Am. A 13, 1698-1703 (1996).

19. L. Cohen, Time-Frequency Analysis (Prentice-Hall, Englewood Cliffs., N.J., 1995); T. A. C. M. Claasen and W. F. G. Mecklenbräuker, "The Wigner distribution: a tool for time-frequency signal analysis," Philips J. Res. 35, 217-250 (1980).

20. A. Walther, "Propagation of the generalized radiance through lenses," J. Opt. Soc. Am. 68, 1606-1610 (1978).

21. D. T. Smithey, M. Beck, M. G. Raymer, and A. Faridani, "Measurement of the Wigner distribution and the density matrix of a light mode using optical homodyne tomography: Application to squeezed states and the vacuum," Phys. Rev. Lett. 70, 1244-1247 (1993). 